\title{
HYPERVELOCITY POWDER DEPOSITION USING PULSED POWER MAGNETIC FLUX COMPRESSION DEVICES
}

\author{
By: \\ M.D. Driga \\ R.C. Zowarka
}

Special Issue of IEEE Transactions on Plasma Science on Pulsed Power Science and Technology (Scheduled for October 2002)

PR - 317

Center for Electromechanics

The University of Texas at Austin

PRC, Mail Code R7000

Austin, TX 78712

(512) 471-4496 


\title{
HYPERVELOCITY POWDER DEPOSITION USING PULSED POWER MAGNETIC FLUX COMPRESSION DEVICES
}

\author{
M. D. Driga \\ Department of Electrical and Computer Engineering \\ The University of Texas at Austin \\ Austin, Texas 78712 U.S.A. \\ driga@mail.utexas.edu \\ (512) 471-6943
}

\author{
R.C. Zowarka \\ Center for Electromechanics \\ The University of Texas at Austin \\ Austin, Texas 78712 U.S.A.
}

\begin{abstract}
This paper outlines two original concepts which, in combination, can bring fundamental advances to both manufacturing and materials technologies and the pulsed power approach to them, and describes a laboratory system already built which has conducted proof-of-principle experiments.
\end{abstract}

The first novel concept uses a hypervelocity accelerator for a new thermal spray process using electromagnetic forces to accelerate power particles to velocities of $2 \mathrm{~km} / \mathrm{s}$ or higher, more than twice that of the powder velocities of about $1 \mathrm{~km} / \mathrm{s}$ used by the existing state-of-the-art thermal spray processes (HVOF, Dgun, plasma spray) which are limited by their reliance on the thermodynamic expansion of gases.

The second original concept is a pulsed power supply, a new electrical machine-flux compressor with precisely controllable output capable of matching ideally the powder spraying hypervelocity accelerator at any moment of time.

Due to the compensation systems, the compulsators are low internal impedance machines designed for pulsed duty and capable of providing large current pulses, with a rapid rise time (in our case, less than $25 \mu$ s to $80 \mathrm{MW}$ pulsed power) to a low impedance load - a hypervelocity square bore railgun accelerator (SBA) assuring an almost continuous process. 
After describing the results of some experiments confirming system modeling and performance, the paper will conclude with future directions of this research.

\section{RESEARCH BACKGROUND AND OBJECTIVES}

The research was based on two original concepts which, in combination, hold the promise of fundamental advances to both manufacturing and materials technologies.

The first novel concept uses a hypervelocity accelerator for a new thermal spray process using electromagnetic forces to accelerate powder particles to velocities of $2 \mathrm{~km} / \mathrm{s}$ or higher [1].

At velocities in excess of $2 \mathrm{~km} / \mathrm{s}$, powder particles have sufficient kinetic energy to melt their own mass and an equivalent substrate mass on impact, thus creating a fusion bond of greater strength than created by the low velocity existing processes, as well as superior coating density and uniformity.

Under an existing contract, proof of principle experiments have confirmed proper operation of the process by capturing photographs of powder particles in flight using state-ofthe-art Hadland CCD framing cameras at The University of Texas at Austin Center for Electromechanics (UT-CEM). The research will now move to operating the process to produce coatings on substrate in an almost continuous mode, requiring a novel, repetitive, pulsed, and extremely powerful power supply, with a high frequency operation, many times a second, day in and day out.

The second original concept is such a power supply, a new electrical machine-flux compressor, pulsed with precisely controllable output capable of matching ideally the powder spraying hypervelocity accelerator at any moment of time.

While novel, a pulsed magnetic flux compressor-rotating electrical machine belongs, in a larger sense, to a family of electrical machines generically named compulsators invented at UT- 
CEM $[1,2,3,4,5,6]$, six of them of very different types which were already built for the U.S. Departments of Defense and Energy. (The U.S. Army is pursuing vigorously the compulsator as the only alternative for power supply for electromagnetic guns, planned to be deployed in the year 2015 [6]).

Due to their compensation systems, the compulsators are low internal impedance machines designed for pulsed duty and capable of providing large current pulses, with a rapid rise time (in our case, less than $25 \mu$ s to $80,000 \mathrm{~kW}-80 \mathrm{MW}$ pulsed power) to a low impedance load-a hypervelocity square bore railgun accelerator (SBA).

The novelty of the proposed machine lies in advancing the use of compulsator and magnetic flux compression principles from the brute force of a pulsed discharge to the refined way of constructing electromagnetically the ideal current pulse shape for the process. As explained in the next section, the proposed machine's generic windings are split into cells and segments, each in series with power electronic devices (IGBTs), forming rotor and stator windings which change their structure during the flux compression.

Successful development of a production prototype will bring fundamental advances to manufacturing. This process can be used to build up material of parent material strength because of the potential to create a fusion bond with the substrate. It can build up the material with less heat input than a welding process, therefore mitigating substrate warpage. The more energetic impact will create denser coatings. It can be used to apply chrome to substrates, therefore avoiding the generation of environmentally hazardous hexavalent chrome, a by-product of electroplating. Due to the improved bond strength, material buildup may be possible, allowing the formation of macrostructures. 


\section{PROCESS FUNDAMENTALS}

The electromagnetic powder deposition (EPD) sprayer, a railgun, consists of two metallic rails with insulating sidewalls separating them. The bore is filled with an ionizable gas, and a radio frequency (rf)-excited cavity at the breech of the accelerator provides a line source of plasma [7]. A high-energy electrical pulse provided by the pulsed energy source (Fig. 1) expands the line source into a planar arc which is driven forward by electromagnetic forces. Fig. 1 shows a simple railgun and the electrical currents and magnetic fields which interact to create the Lorentz force (the cross product of the current density vector and the magnetic field vector). The arc is an efficient snowplow [8], sweeping the gas in the bore to a final velocity approximately twice desired powder velocity. This shocked gas passes over a powder cloud introduced near the end of the gun and accelerates the powder through drag forces. The electrical and powder discharge frequency can be adjusted so that the deposition rate and thermal input to the substrate can be controlled. Considerations of the snowplow mechanism drive by electromagnetic Lorentz railgun forces [9] show that current requirements are related to gas velocity by the relation:

$$
\mathrm{I}=16 \mathrm{~V}_{\text {gas }} \sqrt{\frac{?_{\text {gas }} \mathrm{A}}{\mathrm{L}}}
$$

where the current, $\mathrm{I}$, is in $\mathrm{kA}$, the gas velocity, $\mathrm{V}_{\text {gas }}$, is in $\mathrm{km} / \mathrm{s}, \mathrm{V}_{\text {gas }}$ is the relative density of ambient gas being snowplow accelerated, $\mathrm{A}$ is the cross-sectional area, in $\mathrm{cm}^{2}$ and $\mathrm{L}^{\prime}$ is the inductance of the railgun structure per unit length, in $\mu \mathrm{H} / \mathrm{m}$. For example, using argon at STP (r $=1,389$ times the air value) in a $1.6 \mathrm{~cm}^{2}$ gun structure with inductive gradient $0.5 \mu \mathrm{H} / \mathrm{m}$, to achieve a velocity of $4 \mathrm{~km} / \mathrm{s}$ requires $135 \mathrm{kA}$ driving current.

The electrical pulse width required is dependent on the fraction, $f$, of gas velocity to which the powder particle is to be accelerated. A practical value is $50 \%$ ( $f=0.5$ ). The pulse length is then given by the relation: 


$$
\mathrm{dt}=\frac{4}{3} \frac{?{ }_{\text {powder }}}{?_{\mathrm{gas}}} \frac{\mathrm{D}_{\text {powder }}}{\mathrm{V}_{\mathrm{gas}}}\left(\frac{\mathrm{f}}{1-\mathrm{f}}\right)
$$

where $\delta \mathrm{t}$ is the electrical pulse duration in microseconds, the power source must provide current to the square bore accelerator (SBA) with a rapid rise time $(25 \mu \mathrm{s})$. The ideal current pulse would jump instantly to $150 \mathrm{kA}$, remain constant for about $100 \mu \mathrm{s}$, and drop instantly to $0 \mathrm{kA}$. For laboratory testing, a stage PFN with current waveform (Fig. 2) has been used as a power source.

For the technology to be viable, the process must be driven on a continuous basis. Then the power source must be also an energy storage device capable of multiple shots. Demonstrated energy storage levels listed in [3], in $\mathrm{J} / \mathrm{m}^{3}$, lead us to the electrical machines as a superior choice. However, the $100 \mu$ s pulse width eliminates the ordinary electrical machines from competition due to their extremely low pole pitch.

\section{ROTATING, REPETITIVE POWER SUPPLIES}

We are outlining the features of an original rotating machine, a flux compressorcompulsator-which can efficiently provide $100 \mu$ s pulse width, high current, pulse-shaped, one shot every $30 \mu \mathrm{s}$, approximately 100 million shots per year. The continuous power of the machine is $250 \mathrm{~kW}$ with a pulsed power rating of $80,000 \mathrm{~kW}$ supplied by rapid electromechanical energy conversion of the energy stored kinetically. Fast pulses have been extracted and demonstrated from rotary flux compressors [6,7]. Fig. 3 shows very schematically the capability of the compensated pulsed alternator-flux compressor to deliver large amounts of peak power in less than $1 \mathrm{~ms}$.

Fig. 4 illustrates, as an example, the waveforms for the first compulsator-flux compressor designed and built to power a load of 96 flashlamp circuits used for a laser system. 
Approximately 2.5 MJ of energy and 7.500 MW required by the circuit by the time the current fell to the half-peak value, the half-width of the pulse being $0.325 \mathrm{~ms}$.

Unlike the simplistic principle above, the compulsators in use have a very complex construction, generalizing and exploiting the above principle in a refined and developed manner. In our case, the novel concept of the proposed electrical machine owes the precision of its output characteristics to an original way of controlling the magnetic flux locally in a piecewise manner on both rotors and stators. The winding conductors, stranded and transposed in order to limit the transient field diffusion, are split in elements forming cells and segments, each element being connected in series with fast commutated power electronics devices as IGBTs. Such cells replace the traditional compulsator windings performing the flux compression, by transforming them in windings with variable structure on both rotors and stators controlling (by delay or advance) the flux compression process, thus controlling and synthesizing very precisely the powerful current pulse at each few microseconds interval. The global $80 \mathrm{MW}$ pulse is precisely shaped by local commutation of the cells and segments.

Probably a place where the self-excitation in context of EML technology may be used with spectacular results is the dc series generator, a machine which has no applications in everyday technology, but can serve as the ideal power supply for the augmented railgun used successfully as a hypervelocity plasma sprayer.

The dc series generator, due to its self-excitation particularities, represents an ideal power supply, matching extremely well the characteristics demanded by the hypervelocity augmented railgun plasma accelerator. Closing the generator armature on the practical short-circuit of the railgun starts and leads to self-excitation. 
For such a generator, the no-load characteristic must be calculated as for the separately excited machine, since at no-load, the excitation winding (in series) does not have current. The emf induced in the armature is found with the normal tools and procedures known from the classical electrical machine theory. In this kind of generator (series excitation - self-excited) to every point of the no-load characteristics corresponds a uniquely defined load current flowing through both excitation and armature windings. Fig. 5 shows a connection which will be used in powering the railgun plasma spray device. The self-excitation of both series generators connected parallel is balanced by placing each excitation winding in the armature circuit of the other generator.

Motoring up the generator is done as, of course, a series motor having the advantage of outstanding characteristics of such a drive at an almost $(\mathrm{T} \omega=$ constant $)$ constant power.

\section{NEXT STEP}

The next step for this research tries to complete the path from a promising experiment to a continuous base, electromagnetic powder spray process. The demonstration of the novel concept of electrical machine-magnetic flux compressor as the continuous-duty power supply for the process represents the necessary element for such a path completion. The requirements for the power supply mentioned of $80,000 \mathrm{~kW}$ pulsed power, $250 \mathrm{~kW}$ continuous, came from the energy per pulse of $4.3 \mathrm{~kJ}$ (kinetic energy of the compressed gas and powder $1.18 \mathrm{~kJ}$, resistive energy $2.25 \mathrm{~kJ}$, rail resistive energy $087 \mathrm{~kJ}$ ) in 150,000 A pulses of $100 \mathrm{~s}$ with $25 \mathrm{~s}$ rise time, one pulse every $30 \mathrm{~ms}, 100$ million shots per year.

The methodology of the research will take advantage of a large library of data and the experience of six already built compulsators which have demonstrated with a large safety margin all the global mechanical, electromagnetic, and thermal characteristics of the machine. (The last one, the CCEMG compulsator very recently built, has demonstrated 2,500 MW as compared 
with $80 \mathrm{MW}$ pulsed power required fr the proposed concept of $460 \mathrm{~m} / \mathrm{s}$ peripheral velocity as compared with $340 \mathrm{~m} / \mathrm{s}$ for the flux compressor.) The proposed power supply concept will transform the "compulsator" technology from a very high power pulse approach to the more complex and refined approach in which the power remains large, but is "ideally" controlled by a synthesis process of the variable structure cells achieving the magnetic compression locally and by a subtle strategy microprocessor implemented.

A pulsed transformer will be embedded in the machine, filtering the unwanted output and, by its derivative characteristics, assuring a sudden drop of the current to zero at the end of the shot, thus reclaiming the magnetic energy from the railgun accelerator, and the optimal ending of the pulse.

\section{SUMMARY}

This paper describes a novel hypervelocity power deposition process already proven by proof of principle experiments and a new rotating, pulsed, electrical machine of "active compulsator type" which will make the process highly repeatable, almost continuous. A variant for such a machine is a special self-excited dc series-excited generator.

\section{ACKNOWLEDGEMENT}

The authors wish to acknowledge support for this research under grant ATP 0036580475-2000, administered by the Texas Higher Education Coordinating Board. 


\section{REFERENCES}

[1] J. Bacon, et al, “A New Electromagnetic Powder Deposition System," United Thermal Spray Conference, September 1997, Indianapolis, Indiana.

[2] M.D. Driga, M. Ozdemir, "Compulsators as Pulsed Power Supplies for High-Power, High Energy Powder Spraying," 11th IEEE Internal Pulsed Power Conference, June 29-July 2, 1997, Baltimore, Maryland.

[3] M. D. Driga, "Asynchronous Generators for Fast Pulsed Discharge as Power Supplies for Electromagnetic Launchers," Proceedings of the 9th IEEE International Pulsed Power Conference, Albuquerque, NM, 1993 (K. Prestwitch. W. Baker-Editors), pp. 196-203.

[4] M. D. Driga, "Advanced Concepts for Pulsed Rotating Generators Incorporating Magnetic Flux Compression Features," Proceedings of the 8th IEEE International Pulsed Power Conference, San Diego, CA, 1991, (R. White, K. Prestwitch-editors), pp. 553-557.

[5] W. F. Weldon, M. D. Driga, H. H. Woodson, "Compensated Pulsed Alternator," U.S. Patent 4,200,831, April 29, 1980.

[6] W. F. Weldon, M. D. Driga, S. B. Pratap, "High Energy Pulse Forming Generator," U.S. Patent, 4,935,708, June 19, 1990.

[7] R. Sledge, et al, "Arc Initiation for the Electromagnetic Powder Deposition Gun," United Thermal Spray Conference, September 1997, Indianapolis, Indiana.

[8] J. W. Shearer, et al, "Xenon Shock Waves Driven by High Magnetic Fields," Proceedings 2nd International Conference on Magagauss Magnetic Fields, 1979.

[9] J. Uglum, et al, "Scaling Analysis of the Electromagnetic Powder Deposition Gun," United Thermal Spray Conference, September 1997, Indianapolis, Indiana.

[10] M. D. Driga, S. B. Pratap, W. F. Weldon, "Advanced Compulsator Design," IEEE Transaction on Magnetics, MAG 25, No. 1, January 1989, pp. 142-147. 


\section{FIGURE CAPTIONS}

Figure 1. The plasma armature is accelerated down the length of the railgun by an electromagnetic Lorentz force generated by the interaction of the magnetic field surrounding the rails and the current flowing through the armature.

Figure 2. Gun current generated by the PFN circuit. The peak current and duration are dictated by required powder velocity.

Figure 3. A single armature conductor rotating toward a single compensating conductor. The radial B-field, produced by the stator windings, induces a current in the moving armature conductor which flows through the slip rings and brushes to the load and, subsequently, through the compensating conductor, as shown. As the two conductors are forced together, the compression of the combined fields increases the flux density in the region between them.

Figure 4. Waveforms for a special case to the first compulsator-flux compressor designed to power a load of 96 flashlamp circuits used for a laser system. Approximately $2.5 \mathrm{MJ}$ of energy were to be delivered to the circuit by the time the current fell to the halfpeak value. The lower curves are the dynamic voltage and current during the pulse and the open-circuit voltage when no trigger is fired. The dynamic voltage peaks are higher than the open circuit voltage because of the flux compression effect in the device.

Figure 5. Series excitation pair of dc machines for EML powder deposition. 


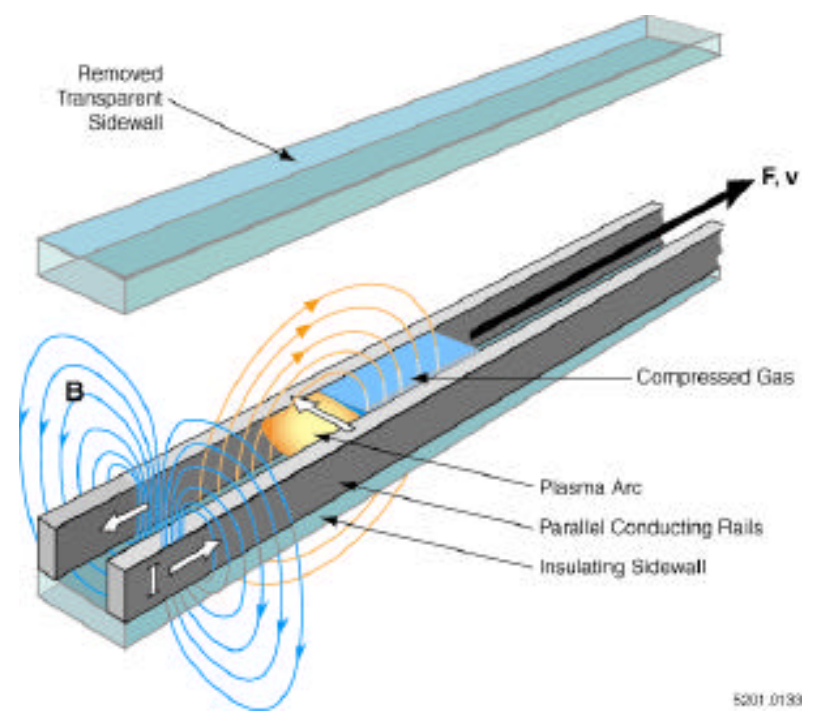

Figure 1 


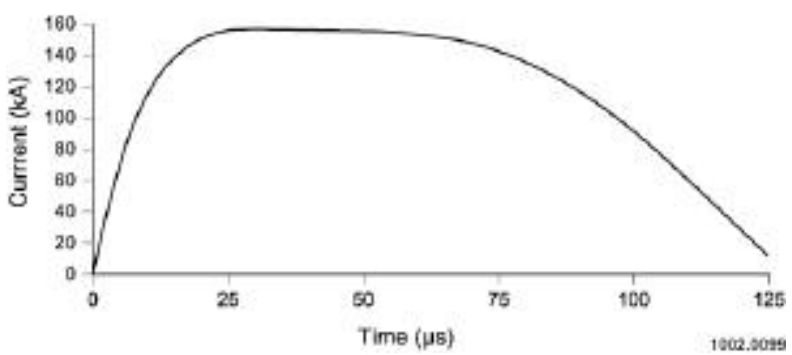

Figure 2 


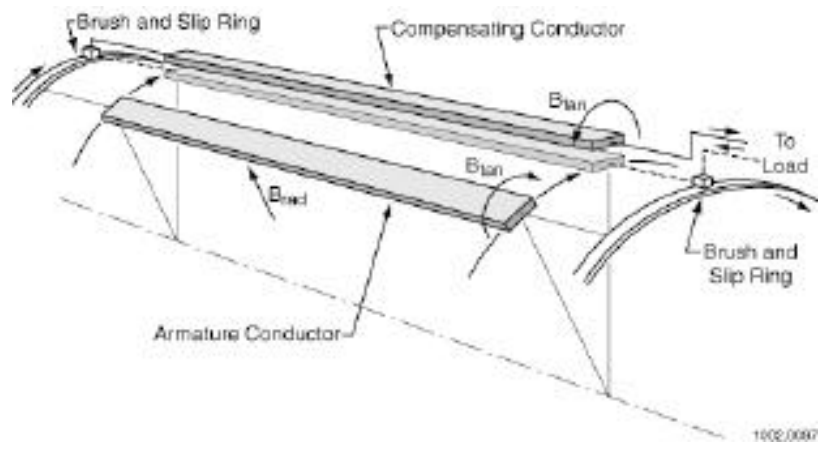

Figure 3 

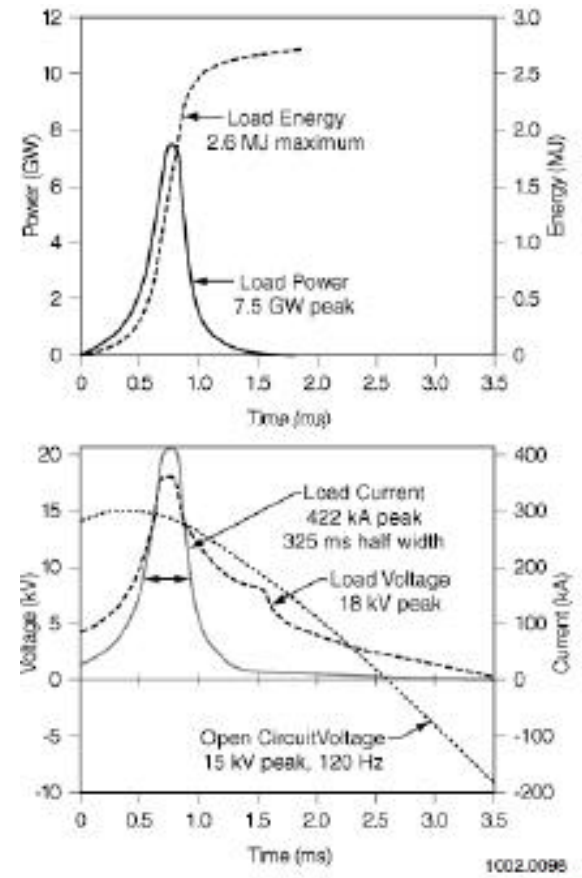

Figure 4 


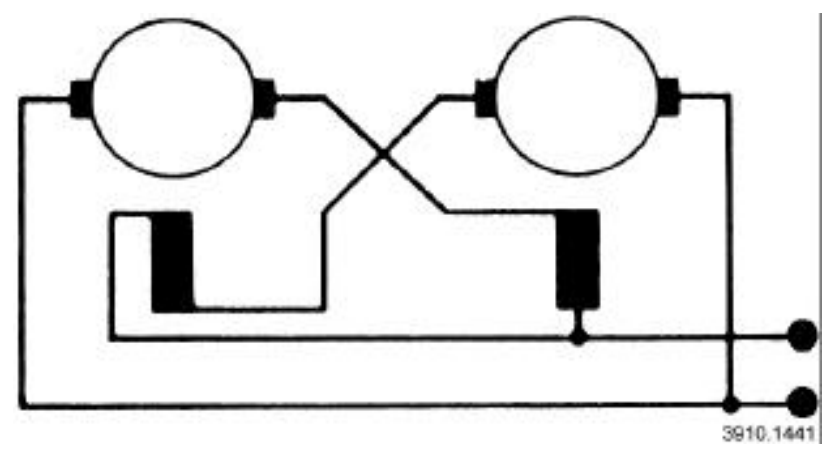

Figure 5 